\title{
INFLUENCIA DEL ESPECTRO SÍSMICO EN LOS EFECTOS DE LOS TERREMOTOS EN LAS EDIFICACIONES
}

\author{
SEISMIC SPECTRUMS INFLUENCE IN BUILDING STRUCTURAL DAMAGE \\ Laura Navas Sánchez , Jaime Cervera Bravo, Belén Benito Oterino \\ Universidad Politécnica de Madrid, Crta de Valencia km 7, 28031, Madrid, España. laura7.I@hotmail.com
}

\begin{abstract}
:
This project aims to analyse the influence that the response spectrum form may have in the damage that a building structure suffers. In order to demonstrate, two different buildings have been modelled: the first represents a typical construction situated in Lorca, which has a long fundamental period. And another which has a short fundamental period, whose singular design allows us to extract some conclusions about the influence of certain factors such as irregularities or torsion effects. Besides, it will be applied in both buildings two different seisms: a short and a long-period response spectrum, so as to study the resonance phenomenon. In addition, it will be exposed briefly the most important changes that have suffered the seismic codes according to the seismic design of building structures.
\end{abstract}

Key words: Seismic Spectrum, Structures, Building, 3D Structural Modelling

\section{Resumen:}

En el presente trabajo se analiza la influencia que puede tener el espectro de respuesta en el daño que una edificación puede sufrir. Para ello, se modelan dos edificios: uno en Lorca, representativo de la tipología estructural del lugar y un edificio singular que, por sus irregularidades, permite extraer conclusiones sobre la influencia del diseño de la estructura en su resistencia frente al sismo. Además, a ambos se los somete a dos espectros sísmicos distintos: uno con un espectro de respuesta de periodo largo, el ocurrido en Mayo de 2011 en Lorca, y otro de periodo corto, el ocurrido en Avezzano, Italia en Octubre de 2016. Finalmente, se incluyen algunos apuntes sobre los avances en materia sísmica de las normativas y su influencia en el diseño antisísmico.

Palabras clave: Espectro sísmico, Estructuras, Edificación, Modelización 3D

\section{Introducción}

En los últimos años los mapas sísmicos de las normativas han sufrido importantes modificaciones. Estas obligarían a algunos edificios ya construidos a sufrir incrementos notables de resistencia para cumplir con los requisitos actuales.

Además, se ha constatado en diversos estudios, que los espectros de respuesta de terremotos recientes, como el ocurrido en Lorca, difieren notablemente de los establecidos por las normativas sismorresistentes españolas (Benito et al. 2012). Tanto las normativas ya derogadas, PDS-1 (1974) y NCSE-94, como la actual, NCSE-02, presentan espectros que dejan a determinados edificios sin la suficiente seguridad.

Puesto que la situación más desfavorable para la estructura es que se produzca el fenómeno de resonancia edificio - sismo, y esto sucede cuando el periodo a fundamental de una estructura está cerca del periodo predominante del movimiento sísmico generado, es necesario realizar el análisis considerando ambos parámetros.

Al mismo tiempo, existe una considerable incertidumbre a la hora de conocer el periodo natural real de una estructura. Los programas de cálculo modernos permiten una buena aproximación, pero dejan de lado factores de considerable influencia, como son los cerramientos 0 incluso las escaleras, que aportan bastante rigidez al sistema real.

El principal objetivo de este trabajo es evaluar la influencia que el contenido frecuencial del movimiento, representado por el espectro de respuesta, puede tener, tanto en un edificio representativo de la arquitectura española de los años 70 , como en un edificio de diseño singular.

Estos se eligen con la intención de que permitan evaluar el efecto de resonancia edificio - sismo ya mencionado; por lo que se opta por un edificio de periodo fundamental corto y otro de periodo largo. Ambos se analizan, con apoyo del programa SAP2000, bajo los efectos de dos sismos también de diferente contenido espectral, uno de periodo predominante corto y otro de largo periodo.

\section{Metodología}

Las normativas sísmicas, desde su aparición, permiten estimar la frecuencia o el periodo natural de vibración de una estructura en función de parámetros como el número de plantas y el material de construcción. 
Sin embargo, en este trabajo se realizará un cálculo más preciso de dicho periodo gracias a las posibilidades de los programas de cálculo actuales. Se pretende de esta forma hallar la respuesta real de los edificios analizados ante los espectros de respuesta de los movimientos considerados, con la máxima precisión posible.

Se seleccionan los siguientes edificios:

- Un edificio residencial de periodo largo, situado en la localidad Lorca, Murcia. Construido en la década de los 70 , cuenta con 5 plantas y un periodo fundamental que supera los 0,5 segundos en cualquier caso (Fig.1).

- La biblioteca pública de Viana Do Castelo, Portugal, proyectada por el arquitecto Álvaro Siza Vieira y construida en el año 2004. Es un edificio de periodo fundamental corto, entorno a $0.1 \mathrm{~s}$, que consta únicamente de planta baja y primera, siendo especialmente singular su irregularidad estructural tanto en planta como en alzado a efectos sísmicos. (Fig. 2)
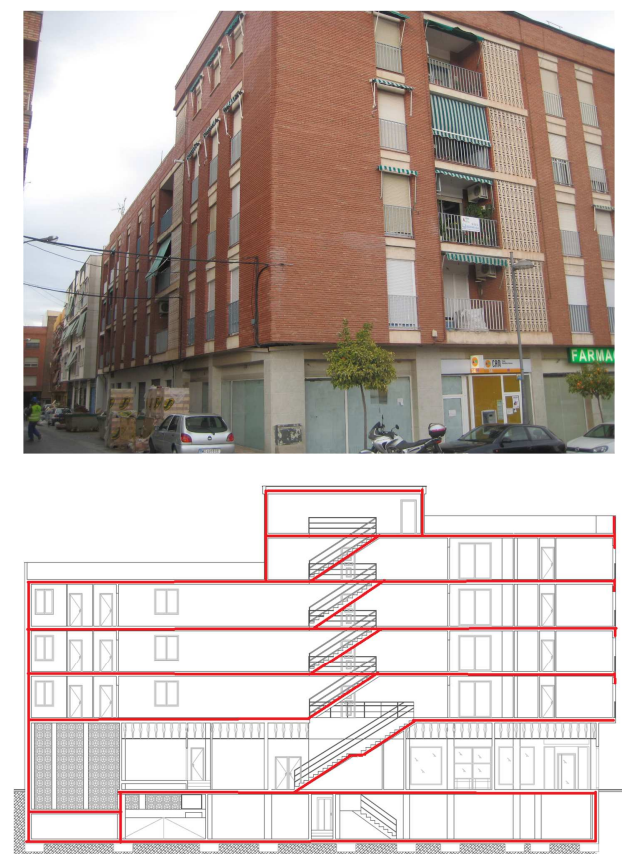

Figura 1: Fotografía y sección transversal del edificio de Lorca.

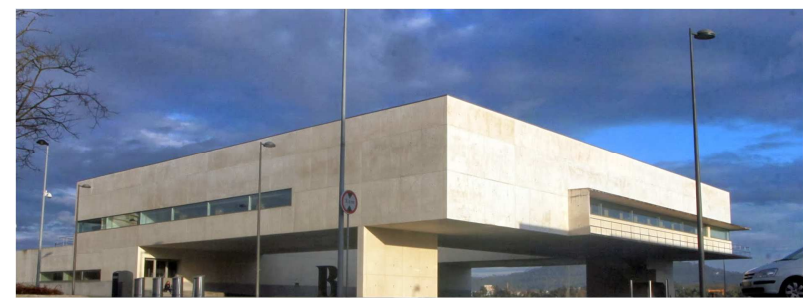

Figura 2: Fotografía de la biblioteca pública de Viana Do Castelo.

Acorde a los edificios seleccionados se eligen los espectros de respuesta de los siguientes movimientos sísmicos (Fig. 3):

- De periodo corto: el espectro de respuesta del terremoto principal que tuvo lugar en la localidad de Lorca, Murcia, el 11 Mayo de 2011. Concretamente se considera el espectro correspondiente al acelerograma registrado en la estación más cercana, en la dirección N30W, que es la máxima componente horizontal.

- De periodo largo: el espectro de respuesta del sismo ocurrido en Avezzano, Italia (30-102016, 6.5 Mw y RAN Station AVZ). Dicho espectro se extrae de la base de datos Center for Engineering Strong Motion Data (CEMD) y se escala con una relación de 0.93 para que los picos de aceleración (PGA) de los dos movimientos considerados sean similares. Esto se realiza con el objetivo de reducir el efecto de la intensidad del movimiento para centrarnos sobre todo en su contenido espectral, es decir en la forma del espectro.

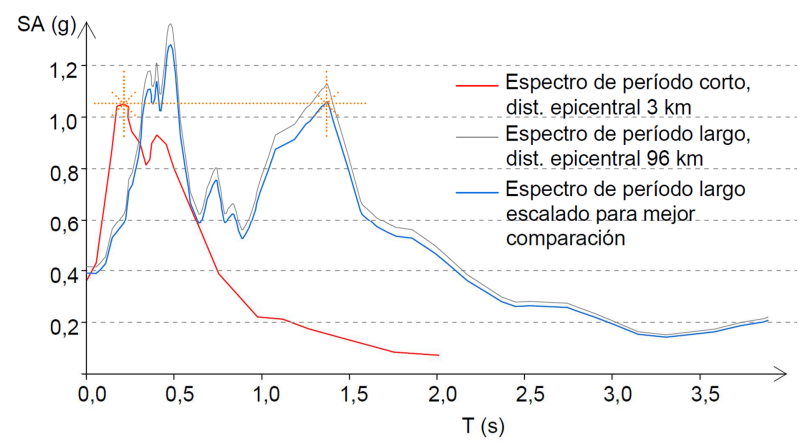

Figura 3: Espectros utilizados para el estudio comparativo.

Mientras el modelado del edificio residencial se realiza con elementos lineales que representan las propiedades de sus soportes y vigas de HA (Fig. 4); para la biblioteca pública de Viana Do Castelo es necesario realizar un modelo de elementos finitos que permita definir su complejo funcionamiento estructural (Navas-Sánchez 2016). Se modela con lajas de hormigón postesado HP50 y muros de hormigón armado HA-50 (Fig. 5). Cabe mencionar que la estructura real esta realizada con acero.

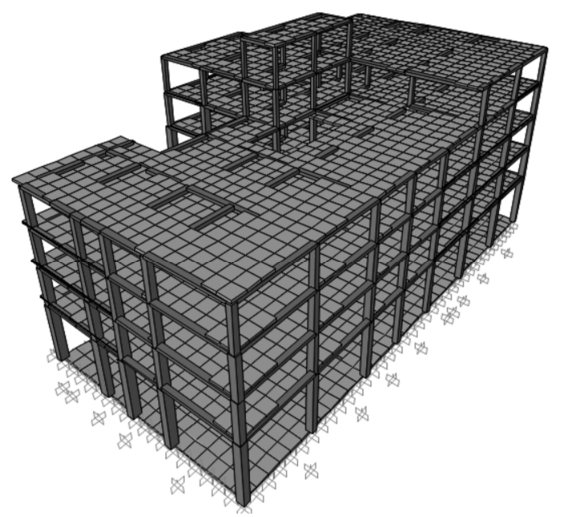

Figura 4: Vista 3D de los modelos introducidos en el programa SAP2000: Edificio residencial en Lorca.

\section{Modos fundamentales de vibración y combinación de modos}

Se observan los modos fundamentales de vibración, es decir, las formas de movimiento de la estructura que requieren menor cantidad de energía para movilizarse 
(Clough and Penzien 2013) que a continuación se describen.

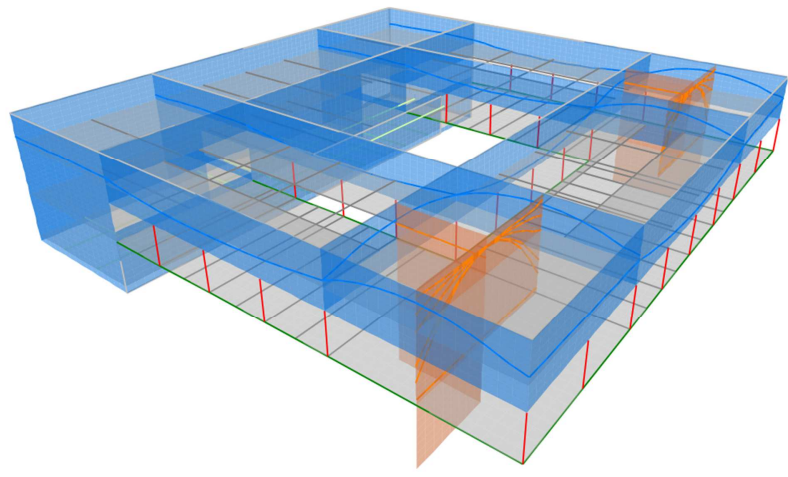

Figura 5: Vista 3D de los modelos introducidos en el programa SAP2000: Biblioteca Viana Do Castelo.

Además, para hallar los esfuerzos y deformaciones resultantes, se realiza en ambos casos una combinación de modos de tipo CQC (Combinación Cuadrática Completa); de forma que se considere el acoplamiento modal entre modos de vibrar parecidos (Wilson 2002).

\subsection{Edificio residencial en Lorca de largo periodo fundamental}

El periodo fundamental T varía según el procedimiento de cálculo utilizado, siendo especialmente relevantes los siguientes resultados:

- Según la Normativa PDS-1, de obligado cumplimiento en el año de construcción del edificio (1978): $\mathrm{T}=0,38 \mathrm{~s}$ (por lo que se toma el mínimo normativo de 0,5 segundos).

- Según la Normativa española vigente, NCSE02: $\mathrm{T}=0,45 \mathrm{~s}$.

- Según la Normativa europea vigente, EN-1998: $\mathrm{T}=0,45 \mathrm{~s}$.

- Según el Modelado con la rigidez bruta en el programa SAP2000: $\mathrm{T}=0,83 \mathrm{~s}$.

- Según el Modelado con la rigidez fisurada, propuesto por EN-1998 en su apartado 4.3.1 (7) en el $50 \%$ de la rigidez bruta: $T=1,22 \mathrm{~s}$. (Fig. 6).

El motivo de considerar rigidez fisurada es la progresiva degradación que sufren algunos materiales al ser sometidos a cargas cíclicas, como puede ser un sismo. El hecho de que el modelado refleje unos periodos señaladamente superiores a los predichos por la normativa revela la influencia en la estimación de la rigidez derivada de la contrastada dificultad de incorporar los elementos secundarios como son las escaleras o los cerramientos y particiones en los modelos habituales en la práctica profesional: como tales elementos no son de relevancia particular en la respuesta resistente, y difíciles de modelar, no se tienen usualmente en cuenta, alterando a la baja la rigidez inicial del edificio real.

Como puede observarse, el modo fundamental implica torsiones, lo que cabe esperar en edificios con posiciones excéntricas en los núcleos de comunicación, aun cuando no se trate de núcleos rígidos, dada la alteración en las dimensiones típicas de estos respecto a las usuales asociadas a los usos principales del edificio.

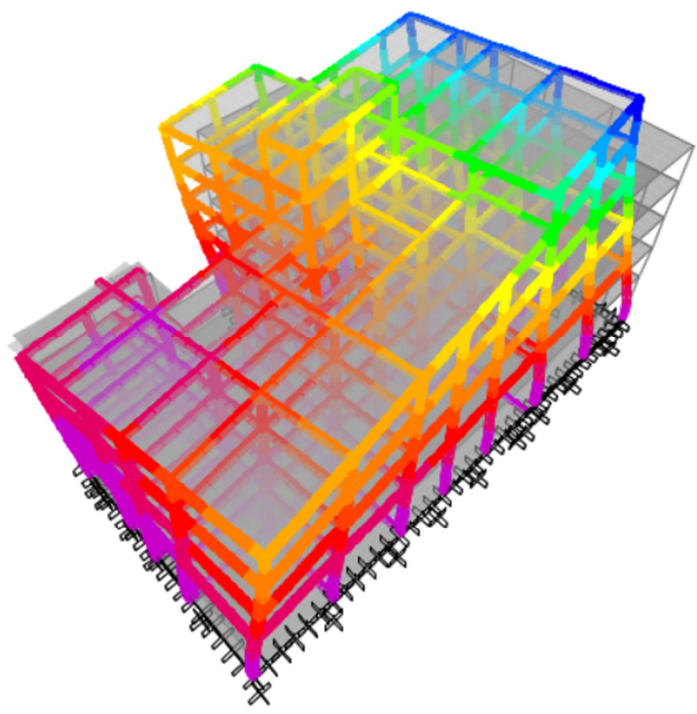

Figura 6: Modo fundamental de vibración del edificio residencial en Lorca. Deformación normalizada por SAP2000 respecto a la situación sin carga (en gris).

\subsection{Biblioteca pública V.Do Castelo de corto periodo fundamental}

En este edificio se observa una importante coincidencia entre el período del modo fundamental de torsión global, calculado mediante el apoyo del programa informático, y el predicho por la norma:

- Según la Normativa española vigente, NCSE02: $\mathrm{T}=0,11 \mathrm{~s}$.

- Según la Normativa europea vigente, EN-1998: $\mathrm{T}=0,13 \mathrm{~s}$.

- Según el Modelado con la rigidez fisurada, propuesto por EN-1998 en su apartado 4.3.1 (7) en el $50 \%$ de la rigidez bruta: $T=\mathbf{0 , 1 4} \mathbf{~ s}$ (Fig. 7).

En este caso el modo fundamental global es de torsión, como se puede observar en la Figura 7. Como era de esperar, la zona apoyada en los muros en $L$ es mucho menos rígida, por lo que los centros de rigidez y de masas están desplazados entre sí y con respecto al centro del cuadrado que define el edificio.

Las normativas desaconsejan este tipo de diseño en zonas sísmicas, debido a que provocan torsión en los elementos estructurales. Sin embargo, este tipo de diseño está presente en un gran número de edificios singulares que se localizan en zonas de media y alta sismicidad en España; como puede ser el palacio de exposiciones y congresos de Granada o el Proyecto de Centro Tecnológico de la Madera en Lucena, Córdoba.

Al ser un elemento de poca altura y con grandes muros de hormigón, a pesar de su singular geometría posee un periodo similar a los edificios regulares de la misma altura. 

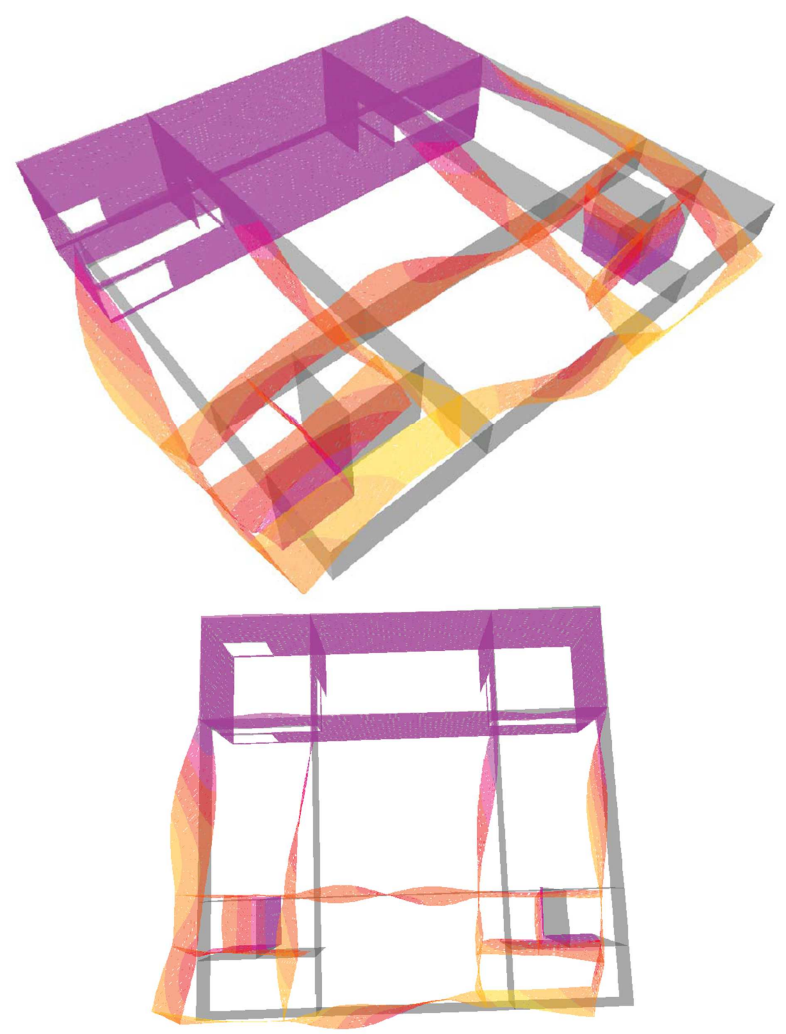

Figura 7: Modo fundamental de vibración de la Biblioteca Pública de Viana Do Castelo. Deformación normalizada por SAP2000 respecto a la situación sin carga (en gris).

\section{Esfuerzos y deformaciones resultantes}

Se realiza el análisis modal espectral pertinente con apoyo del programa SAP2000 y se resumen los resultados más representativos para cada tipo de edificio y movimiento analizados.

Los espectros se aplican en dos direcciones:

- D1. En la dirección que provocan mayor efecto se considera el $100 \%$ del espectro.

- D2. En la dirección perpendicular a la anterior se considera el $30 \%$ del espectro.

De esta forma tratamos de aproximarnos en la mayor medida posible a los criterios que define la normativa actual.

\subsection{Edificio residencial en Lorca de largo periodo fundamental}

En este edificio se decide elegir un pilar de planta baja para comparar resultados, por ser los elementos con mayor probabilidad de fallo debido a la concentración de esfuerzos.

\subsubsection{Esfuerzos principales}

Al aplicar los espectros definidos en las dos direcciones anteriores se obtiene la envolvente de los máximos esfuerzos resultantes con cada espectro. Las Figuras 8 ,9 y 10 muestran las envolventes de los esfuerzos resultantes en el caso más desfavorable.

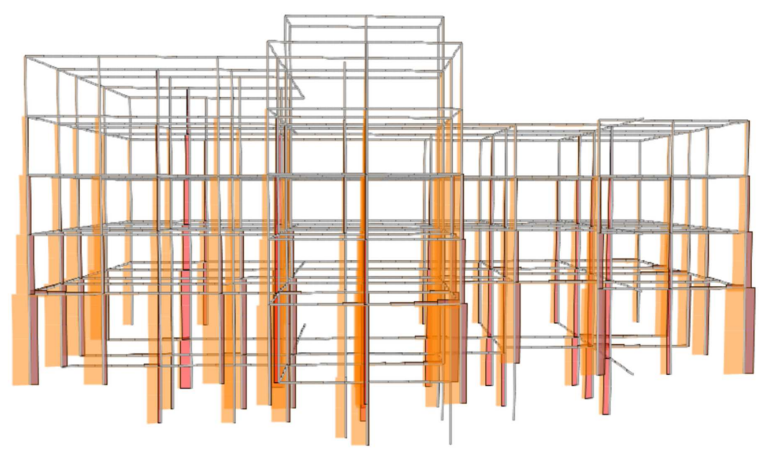

Figura 8: Envolvente del esfuerzo axil en la estructura.

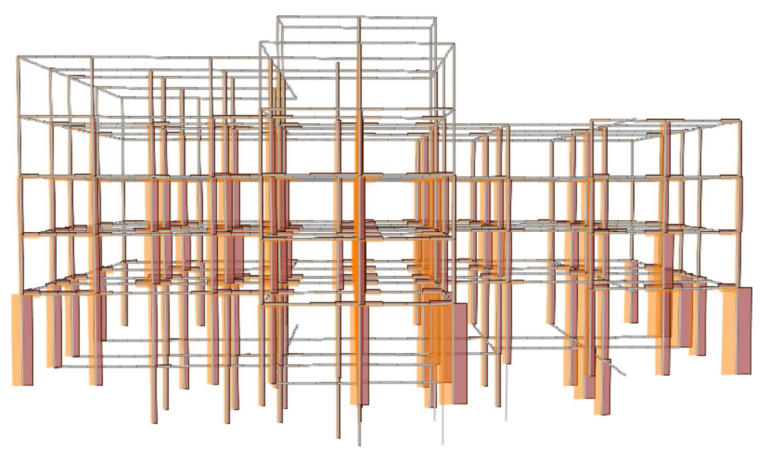

Figura 9: Envolvente de esfuerzo cortante de la estructura en la dirección D1.

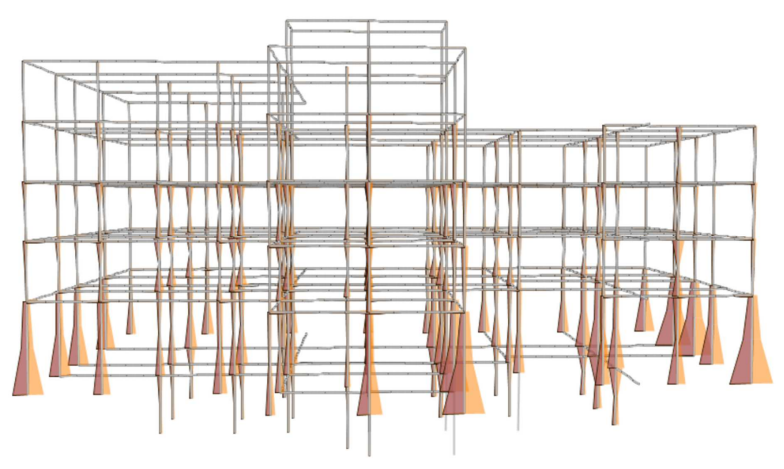

Figura 10: Envolvente de momento flector en la dirección D1.

\subsubsection{Deformaciones}

\subsubsection{Espectro de periodo corto}

El desplazamiento máximo obtenido como resultado de la combinación de modos se produce en las plantas altas y alcanza el valor de $105 \mathrm{~mm}$ (Fig. 11).

Por tanto el desplome se sitúa en valores en torno al 6 por mil, mayor al 5 por mil, definido por EN-1998 como aconsejable.

\subsubsection{Espectro de periodo largo}

El desplazamiento máximo alcanza un valor de $420 \mathrm{~mm}$, muy superior a la resultante del espectro de periodo corto (Fig. 12). En este caso el edificio estaría mucho más cerca del colapso. 


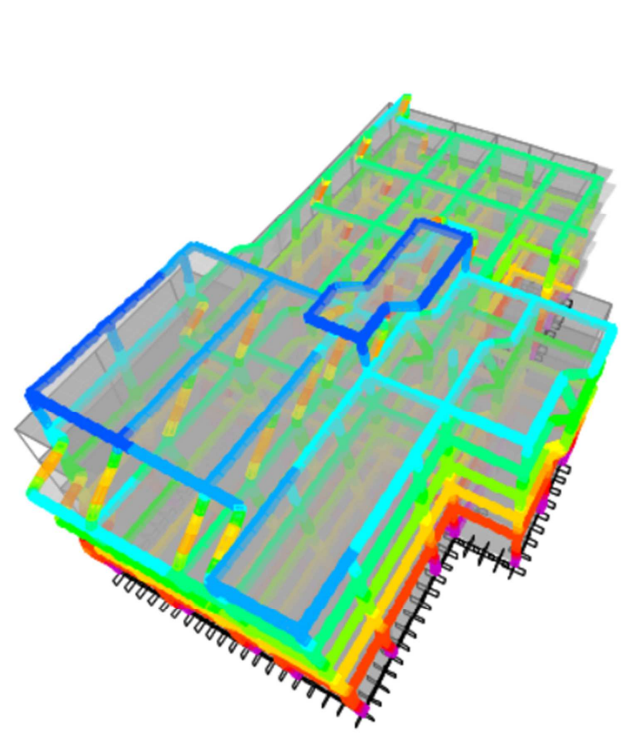

Figura 11: Deformada de la estructura (mm).
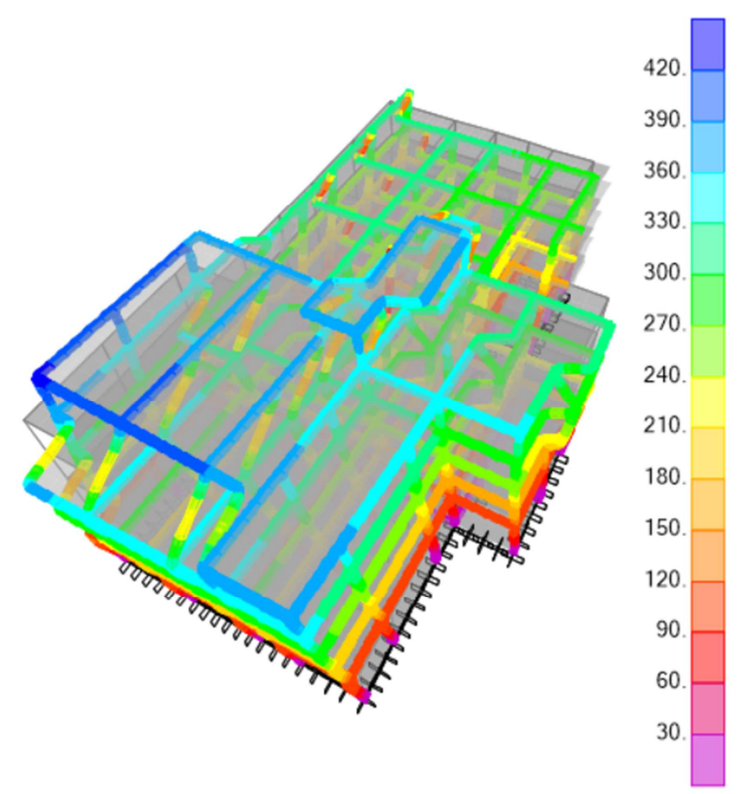

Figura 12: Deformada de la estructura $(\mathrm{mm})$.

\subsubsection{Comparativa}

Considerando una masa total en combinación sísmica de 2060 toneladas se extraen los siguientes resultados.

\subsubsection{Esfuerzos y deformaciones globales}

Con el objetivo de evaluar la diferencia en la respuesta de la estructura, debido tanto a la forma como a la amplitud del espectro, se adjuntan las siguientes tablas de datos extraídos del análisis modal espectral realizado (Tablas 1 y 2 ):

\subsubsection{Esfuerzos y deformaciones locales}

Se analizan los esfuerzos y deformaciones del soporte señalado en la Figura 13. En concreto se dan los valores máximos, que ocurren en la planta baja.
Tabla 1: Desplazamientos y cortantes basales máximos para un T natural de la estructura de 1,22 s. Dirección principal del espectro: D1

\begin{tabular}{c|c|c} 
& $\begin{array}{c}\text { Espectro } \\
\text { periodo corto }\end{array}$ & $\begin{array}{c}\text { Espectro } \\
\text { periodo largo }\end{array}$ \\
\hline $\begin{array}{c}\text { Cortante basal dirección } \\
\text { D1 (kN) }\end{array}$ & 3090 & 11290 \\
$\begin{array}{c}\text { Cortante basal dirección } \\
\text { D2 (kN) }\end{array}$ & 1020 & 4045 \\
$\begin{array}{c}\text { Desplazamiento máximo } \\
(\mathrm{mm})\end{array}$ & 105 & 420 \\
Aceleración aplicada (/g) & 0.19 & 0.92
\end{tabular}

Tabla 2: Esfuerzos principales para el mismo soporte de la estructura

\begin{tabular}{c|c|c} 
& $\begin{array}{c}\text { Espectro } \\
\text { periodo corto }\end{array}$ & $\begin{array}{c}\text { Espectro } \\
\text { periodo largo }\end{array}$ \\
\hline $\begin{array}{c}\text { Axil máximo }(\mathrm{kN}) \\
\text { Cortante máximo en D1 } \\
(\mathrm{kN})\end{array}$ & 325 & 755 \\
$\begin{array}{c}\text { Momento flector } \\
\text { máximo en D1 }(\mathrm{kN})\end{array}$ & 350 & 350 \\
& & 1285
\end{tabular}

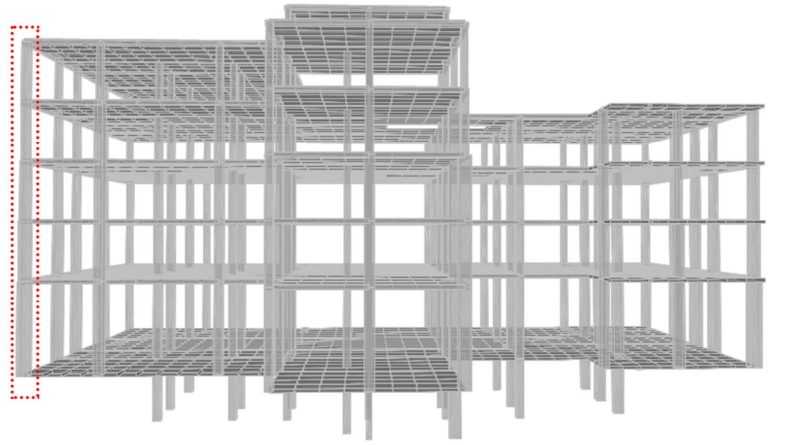

Figura 13: Soporte elegido para la comparación

\subsection{Biblioteca pública V. Do Castelo de corto período fundamental}

En este caso se analizan los esfuerzos resultantes en el punto más conflictivo de la estructura, los muros en L, ya que el sismo transmite al edificio una fuerza principalmente de cortante, y estos son los elementos menos redundantes de la estructura.

\subsubsection{Esfuerzos principales}

Se obtienen los resultados que se muestran en las Figura 14, (se representan de forma adimensional las envolventes de esfuerzos referidas al mayor de los valores de cada tipo para ambos espectros):

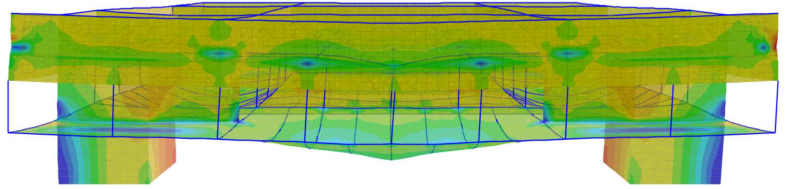

Figura 14: Tensiones S22 sobre la estructura deformada por el sismo (adimensional). 


\subsubsection{Deformaciones}

Se obtienen los siguientes resultados.

\subsubsection{Espectro de periodo corto}

El desplazamiento lateral es muy bajo en cualquier caso, unos $5 \mathrm{~mm}$, debido a la elevada rigidez de la estructura (Fig. 15).
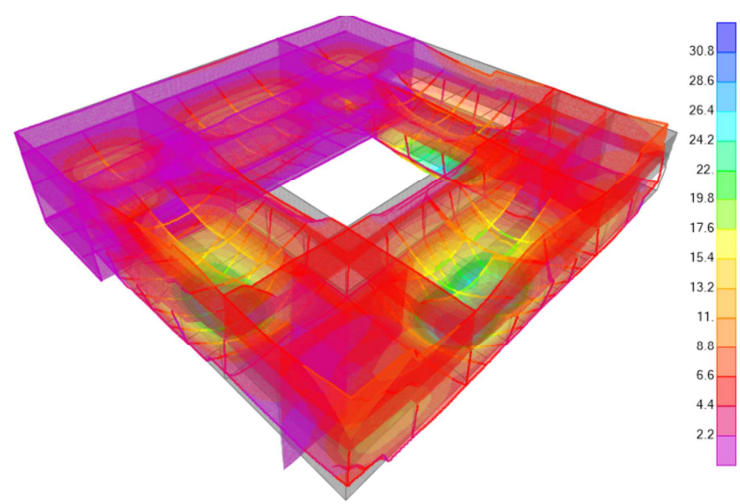

Figura 15: Deformada de la estructura (valores en $\mathrm{mm}$ ).

\subsubsection{Espectro de periodo largo}

De nuevo, el desplazamiento es muy pequeño: unos $5 \mathrm{~mm}$ (Fig. 16).

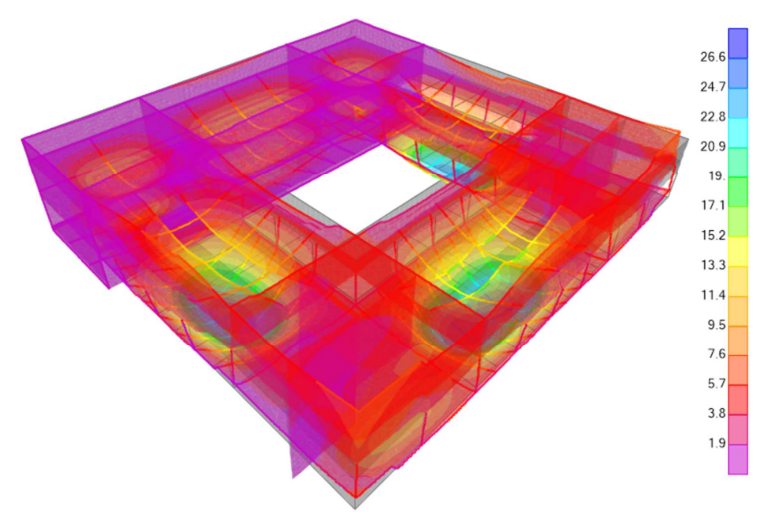

Figura 16: Deformada de la estructura (valores en $\mathrm{mm}$ ).

\subsubsection{Comparativa}

Considerando una masa total en combinación sísmica de 5360 toneladas se extraen los siguientes resultados:

\subsubsection{Esfuerzos y deformaciones globales}

Con el objetivo de evaluar la diferencia en la respuesta de la estructura, debido tanto a la forma como a la amplitud del espectro, se adjuntan las siguientes tablas de datos extraídos del análisis modal espectral realizado (Tablas 3 y 4 ):

\subsubsection{Esfuerzos y deformaciones locales}

Se analizan los esfuerzos y deformaciones locales en los elementos más solicitados: los muros en L.
Tabla 3: Desplazamientos de la estructura de 1,22 s. Dirección principal del espectro: D1

\begin{tabular}{c|c|c} 
& $\begin{array}{c}\text { Espectro } \\
\text { periodo corto }\end{array}$ & $\begin{array}{c}\text { Espectro } \\
\text { periodo largo }\end{array}$ \\
\hline $\begin{array}{c}\text { Cortante basal dirección X } \\
(\mathrm{kN})\end{array}$ & 10015 & 6470 \\
$\begin{array}{c}\text { Cortante basal dirección Y } \\
(\mathrm{kN})\end{array}$ & 5040 & 3870 \\
$\begin{array}{c}\text { Deform. Máxima }(\mathrm{mm}) \\
\text { Aceleración aplicada }(/ \mathrm{g})\end{array}$ & 5 & 5 \\
& 0.61 & 0.42
\end{tabular}

Tabla 4: Esfuerzos principales para un muro en $L$ de la estructura

\begin{tabular}{c|c|c} 
& $\begin{array}{c}\text { Espectro } \\
\text { periodo corto }\end{array}$ & $\begin{array}{c}\text { Espectro } \\
\text { periodo largo }\end{array}$ \\
\hline Axil máximo (kN) & 3735 & 2625 \\
$\begin{array}{c}\text { Cortante máximo en D1 } \\
(\mathrm{kN})\end{array}$ & 4000 & 2375
\end{tabular}

\section{Conclusiones}

De los resultados obtenidos, en términos de esfuerzos y respuesta de las estructuras, se pueden extraer las siguientes conclusiones:

\subsection{Relevancia de la forma espectral}

Se puede concluir que una buena caracterización del espectro de respuesta del movimiento sísmico tiene una altísima repercusión en el diseño antisísmico de edificios. El hecho de utilizar en los cálculos de la estructura espectros de diseño menores que los de los movimientos que puedan producirse (terremotos reales) puede llevar a importantes daños materiales y sobre todo a víctimas humanas.

Conocer con mayor precisión las frecuencias o contenido espectral de los movimientos que pueden esperarse en una cierta localidad es indispensable para mejorar las prácticas constructivas de la zona y proteger los edificios ya construidos.

En los casos analizados, en un mismo edificio puede ser recomendable elevar o disminuir la rigidez/flexibilidad de una estructura en función de la forma espectral del posible evento sísmico concreto que se vaya a dar en el lugar.

Además, como se deriva de los valores de esfuerzos obtenidos en el edificio residencial de Lorca, de largo periodo, el hecho de que el sismo tuviese un periodo espectral relativamente corto en relación al del edificio, debido a su cercanía a la falla que originó el terremoto, contribuye en gran medida a que el edificio resista el evento al que se ve sometido.

\subsection{Diseño estructural sismorresistente}

Para conseguir un buen diseño estructural sismorresistente se ha de buscar:

- La máxima simetría posible tanto en planta como en alzado. Recurriendo, si es necesario, a la colocación de juntas de dilatación entre cuerpos regulares independientes. 
- La mínima masa posible, para así reducir las fuerzas que provoca el sismo en la estructuras.

- La rigidez que confiera al edificio un periodo lo más alejado posible del periodo predominante del movimiento sísmico.

- Asimismo, evitar plantas con diferente rigidez en el mismo edificio.

- Proyectar estructuras dúctiles, para que la energía se disipe por medio de deformaciones plásticas remanentes, sin llegar al colapso.

- Evitar puntos débiles como nudos con rotura frágil, especialmente la rotura por cortante en elementos de hormigón armado.

- Procurar la redundancia de elementos primarios, cuyo fallo conduciría al colapso global de la estructura, como ocurriría en la biblioteca de Viana Do Castelo en el caso de fallar los muros en $\mathrm{L}$.

\subsection{Desarrollo de la normativa}

La normativa vigente en el momento de construcción del edificio residencial en Lorca (PDS-1) evidencia importantes carencias a la hora de caracterizar el terremoto de diseño. Para calcular las fuerzas aplicadas no se considera el espectro real del (único) terremoto de referencia para el proyecto, sino únicamente su posible intensidad en la escala internacional macrosísmica (M.S.K.), y un factor de respuesta $\beta$ dependiente del periodo de oscilación natural de la edificación considerada. La relación entre ambos sería la siguiente, Ec. (1):

$\beta=B /(2 T) \geq 0.5$

dónde:

- $T=$ periodo fundamental estimado de la estructura

- $\quad B=$ amortiguamiento característico del edificio

Análogamente, la norma NCSE-02 sólo considera la posible alteración en la forma de los espectros de respuesta dentro del territorio español para el terremoto de referencia a través de un factor $K$ (de carácter geográfico) y del coeficiente del suelo $C$ (de carácter geotécnico), con reducida capacidad en la precisión de los diferentes espectros esperables.

Además, cabría añadir como recomendación para la revisión de la normativa sismorresistente vigente que se haga explícito que, en caso de no incluir en el modelado los elementos secundarios en términos de rigidez, se considere para la rigidez inicial un valor apreciablemente mayor (entorno a un $50 \%$ ) que la calculada por los programas comerciales.

Las normativas que se están desarrollando en este momento en Europa, condicionan los esfuerzos resultantes a la forma del espectro de respuesta del movimiento sísmico, mediante un análisis modal espectral similar al realizado en este trabajo. Además, incluyen un nuevo concepto: el proyecto basado en prestaciones, cuyo objetivo es producir estructuras con un comportamiento sísmico predecible, ajustado a cada tipo de movimiento esperable (corto o largo período) en función de la probabilidad de alcanzar cada posible intensidad (Benavent 2017).

Este proyecto permite que la respuesta del edificio ante cada posible movimiento se encuentre en uno de los estados definidos dentro de un abanico de respuestas estándar preestablecidas: 1) "operacional", 2) "es posible la ocupación inmediata", 3) "se producen daños controlados", 4) "se asegura la protección de la vida", 5) "se mantiene la estabilidad estructural", etc.

El estado permitido para el edificio depende de su utilidad e importancia. Por ejemplo, un hospital debe mantenerse "operacional", mientras que una vivienda convencional debe "asegurar la protección de la vida".

En este trabajo hemos comprobado que los diferentes movimientos sísmicos que pueden esperarse en un cierto emplazamiento condicionan tanto la forma como la amplitud de los espectros de respuesta, y ello, a su vez tiene influencia decisiva en la respuesta de edificios dependiendo de sus cualidades sísmicas. Estas deberán adaptarse tanto a la intensidad del movimiento como a su contenido espectral.

\section{Agradecimientos}

Este trabajo ha sido financiado por el Proyecto MERISUR, Programa Estatal Español de I+D+I Orientada a los Retos de la Sociedad, Ministerio de Economía (ref. CGL2013-40492-R).

\section{References}

BENAVENT CLIMENT, A., 2017. Estrategias convencionales y avanzadas de proyecto sismorresistente, Conferencia en el Máster de Estructuras de la Edificación, Ciclo de conferencias en la Escuela Técnica Superior de Arquitectura, Universidad Politécnica de Madrid.

BENITO OTERINO, B., RIVAS MEDINA, A., GASPAR-ESCRIBANO, J.M. and MURPHY, P., 2012. El terremoto de Lorca (2011) en el contexto de la peligrosidad y el riesgo sísmico en Murcia. Física de la Tierra, 24, pp. 255-287.

CENTER FOR ENGINEERING STRONG MOTION DATA, 216. Northern Norcia Italy Earthquake Of 30 Oct 2016. Available: https://www.strongmotioncenter.org/cgi-bin/CESMD/Multiplesearch1_DM2.pl?event_name=\&magmin= \&magmax $=\&$ byear $=\&$ eyear $=\&$ country $=A n y \& s t a t e=A n y \& s t n \_i d e n t=A v e z z a n o \& t y p e=A n y \& M a t e r i a l=A n y \& H e i g h t=\& a c c$ $\min =\& a c c m a x=\&$ hdistmin $=\&$ hdistmax $[4 / 25,2017]$.

Clough, R. W. and PENZIEN, J., 2003. Dynamics Of Structures, Computers \& Structures, Inc., Third Edition. 1995 University Ave., Berkeley, CA 94704, USA.

NAVAS SÁNCHEZ, L., 2016 Biblioteca municipal: Viana do Castelo (Portugal), Álvaro Siza Vieira : [Cálculo de la estructura en zona sísmica], Master Thesis, Superior Technical School of Architecture, Polytechnic University of Madrid. Available: http://oa.upm.es/44505/. 
NCSE-94, 1994, Norma de Construcción Sismorresistente: Parte General y Edificación, Real Decreto 2543/1994, 29 de Diciembre.

NCSE-02, 2002, Norma de Construcción Sismorresistente: Parte General y Edificación, Real Decreto 997/2002, 27 de Septiembre.

P.D.S.-1, 1974, Norma Sismorresistente, Real Decreto 3200/1974, 30 de Agosto.

WILSON, E.L., 2002. Three-Dimensional Static and Dynamic Analysis of Structures, A Physical Approach With Emphasis on Earthquake Engineering, Third Edition, Computers and Structures, Inc., Berkeley, California, USA, ISBN 0-923907-00-9. 